\title{
The role of psychological interventions in chronic headache management: a case report
}

Rosario Iannacchero ${ }^{1}$, Alessandra Sansalone ${ }^{1}$, Amerigo Costa ${ }^{1}$,Ermanno Pisani ${ }^{2}$, Antonio Siniscalchi ${ }^{2}$

\begin{abstract}
According to the biopsychosocial model, biological, psychological, and social factors entwine and influence each other bi-directionally concerning health conditions. Therefore, especially in disorders such as chronic headache, where behavioral and environmental factors are prominent risk factors and triggers, psychosocial interventions might contribute to reduce the burden of the bealth condition and related affective disorders and disability.

We present the case of a Medication Overuse Headache patient self-reporting psychosocial and cognitive issues, that prompted further clinical-psychological and neuro-cognitive assessment and eventually psychological interventions targeted at headache management in conjunction with medical treatment. Psychological interventions were tailored to the patient's features and presentation and were successful in reducing headache attacks in terms of intensity, frequency, and debilitation, thereby resulting also in a better perceived general health.
\end{abstract}

Keywords: Headache Disorders; Migraine Disorders; Psychology; Medication Overuse Headache

Il ruolo degli interventi psicologici nella gestione del mal di testa cronico: un caso clinico CMI 2017; 11(1): 1-5

http://dx.doi.org/10.7175/cmi.v11i1.1287

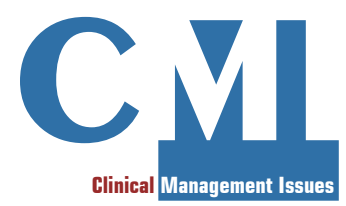

Case report
${ }^{1}$ Centre for Headache and Adaptive Disorders, Unit of Neurology, Department of Neuroscience and Sensory Organs, "Pugliese - Ciaccio" Hospital,

Catanzaro, Italy

${ }^{2}$ Unit of Neurology, Department of

Neuroscience and Sensory Organs, "Dell'Annunziata" Hospital, Cosenza, Italy

\section{INTRODUCTION}

Chronic Daily Headache (CDH) is among the most frequent and disabling neurological conditions, affecting $4 \%$ to $5 \%$ of the general population [1] and being one of the main causes of disability for the adults [2]. $\mathrm{CDH}$ is a category comprising different disorders, all of them presenting with headache attacks occurring at least 15 days a month [1]. A large burden comes from Chronic Migraine (CM), which affects approximatively $2 \%$ of the general population [3] and consists of migraine attacks occurring at least 15 days per month [4].

Treatment of $\mathrm{CDH}$ usually involves preventative therapy to be taken daily in order to prevent attacks, and attack therapy to be taken every time a headache attack starts [5]. Common preventative headache drugs are, for instance, anticonvulsants and antidepres- sants, while nonsteroidal anti-inflammatory drugs (NSAIDs), other pain relievers and headache-specific triptans are used, among

\section{Why do we describe this case}

We describe this case to underline that chronic headache can be successfully addressed using a biopsychosocial framework. Tailored psychological interventions in conjunction with medical therapy might be able to enhance clinical and psychosocial outcomes. Many headache triggering or interfering with headache and its management factors are grounded on a behavioral level, then psychological interventions might help removing some obstacles to reaching satisfactory health
Corresponding author Rosario Iannacchero centrocefaleeaopc@gmail.com

Disclosure

The authors declare they have no competing financial interests concerning the topics of this article 
others, as attack treatments [5]. Another preventative pharmacological option for $\mathrm{CM}$ consists of periodical botulinum toxin injections [6].

Medication Overuse Headache (MOH) is a CDH caused by the patient's excessive use of pain medications [4]. It is a disabling condition rooted in a compulsive behavior and it might need a complex setting of care in order to be effectively treated: lack of control in regards to medication usage and other co-existing behavioral problems need to be addressed [7]. There is a high prevalence of mental health problems among $\mathrm{CDH}$ sufferers, that can constitute an obstacle to an effective management of the condition [8]. Chronic headache disorders might then need a comprehensive approach with treatments aimed not only at reducing frequency and severity of the headache episodes, but also at decreasing the effects of headache on psychosocial functioning [9]. Clinicians may want to consider all factors co-determining disorder onset, progression, severity, and disability in order to provide effective care [9].

In later decades, a theoretical model underlining the interaction of physiological, psychological (cognitive, behavioral, and affective) and social (environmental, work, and family) factors has emerged as a comprehensive model of understanding and treating chronic headache, the biopsychosocial model [10]. According to this model, biological, psychological, and social factors entwine and bi-directionally influence each other [10]. Therefore, including psychosocial principles and interventions in a setting of care might contribute to reduce health condition burden and related psychosocial problems [9]. The most common reason for referring a headache patient to a clinical psychologist, a counseling psychologist, or a psychotherapist is the presence of major mental health and behavioral disorders like mood disorders, anxiety disorders, sleep disorder, eating disorders, and addictions [9]. Other reasons for a psychological referral are cognitive and behavioral elements related to ineffective management of the health condition such as poor trigger management, poor stress management, and excessive use of medications [9].

Various methods of psychological intervention are available in headache and pain management [11] and we present a case of a chronic headache with excessive use of medications treated, according to the biopsychosocial model, with medical therapy and supportive psychological interventions tailored to the patient's features and presentation.

\section{CASE DESCRIPTION}

The patient is a female 52-year-old homemaker, married and living with her husband and mother, who was referred by her general practitioner and came to the Centre for Headache in the fall of 2014 (T1). She had a 15-year clinical history of chronic migraine (CM) and no other significant medical condition. She also reported high level of stress, feeling the burden of her homemaker and caregiver duties and reported anxious symptoms. During the previous 15 years, she had a semi-continuous use of different NSAIDs, mostly as self-medication, such as ibuprofen, ketoprofen, and recently diclofenac $50 \mathrm{mg}$ (she would occasionally take up to $150 \mathrm{mg}$ in a day). She reported having used under prescription $100 \mathrm{mg}$ of topiramate per day as a preventative therapy years ago without satisfactory results. She referred pulsating and stabbing headache attacks located in left and right (not simultaneously) temporal and orbital head areas. She reported 30 Headache Days Per Month (HDPM). She would rate 9 out of 10 her usual headaches on the pain Numerical Rating Scale (NRS). The attacks would last from 4 to 12 hours. She had often nausea, photophobia, and phonofobia during attacks. The neurological examination had otherwise normal findings.

Brain magnetic resonance imaging (MRI) showed an occipital arachnoid cyst that was considered being likely asymptomatic. Venous magnetic resonance angiography (vMRA) was negative. At the psychological evaluation, the patient reported significant levels of anxiety and cognitive issues, especially regarding memory, attention, and focusing. The patient completed questionnaires regarding anxiety (Zung Self-Rating Anxiety Scale $=45$; normal values: $20-40$ ), depression (Zung Self-Rating Depression Scale $=38$; n.v. $=20-43)$, cognitive functioning (Cognitive Failure Questionnaire = 36; n.v. $=0-42$ ) and disability (Migraine Disability Assessment - MIDAS = 56; n.v. $<21$ ).

The patient was diagnosed probable Medication Overuse Headache $(\mathrm{pMOH})$ and was prescribed levetiracetam ( $250 \mathrm{mg}$ to 500 $\mathrm{mg}$ per day) as a preventative therapy and almotriptan $(12.5 \mathrm{mg})$ as an attack therapy. In addition, the patient was advised to avoid 
taking NSAIDs for the headache and to compile a headache diary.

After three months (T2), headache frequency, intensity, and disability were slightly reduced (25 HDPM; NRS = 8; MIDAS = 48). Examining the headache diary, it emerged that adherence to therapy was partial and NSAIDs were still used albeit in a reduced scale. The $\mathrm{MOH}$ diagnosis was confirmed. The patient kept reporting her psychosocial issues. Even though no major emotional or cognitive impairment was evident at the interview, a more comprehensive neuro-cognitive and clinical-psychological assessment was performed. General cognitive efficiency, memory, constructional praxis, and executive functions were assessed. Personality was assessed using Minnesota Multiphasic Personality Inventory (MMPI-2), that showed no psychopathological scores, and Barratt Impulsiveness Scale (BIS-11), that showed mild personal impulsivity. The neuropsychological profile was borderline regarding general cognitive efficiency, abstract logical reasoning, prose memory, semantic fluency, spatial planning, and attention. Performance deficits were detected in visual short-term memory, verbal long-term memory, visual long-term memory, phonemic fluency. These findings were not sufficient for a neuro-cognitive or psychopathological diagnosis, but they had nonetheless psychometric value in terms of patient's understanding. It was hypothesized that a probable executive impairment could relate with behavioral aspects relevant to $\mathrm{MOH}$ and chronic headache, such as non-adherence and compulsive medication use [12].

Considering that the patient could benefit from psychological and educational interventions in conjunction to the medical therapy, she was proposed to attend a number of 6 psychological sessions during the following 2 months. The patient was advised to continue medical therapy as prescribed before. During the psychological sessions some cognitive, behavioral, affective, and personality elements relevant to headache management were addressed. Initially, using diaries, headache triggers were identified and their relationship with affective states of anxiety, anger, frustration, and depletion of mood was explored. Health behaviors and adherence to medication usage were emphasized with a focus on personal ability to actively pursue headache management with effective

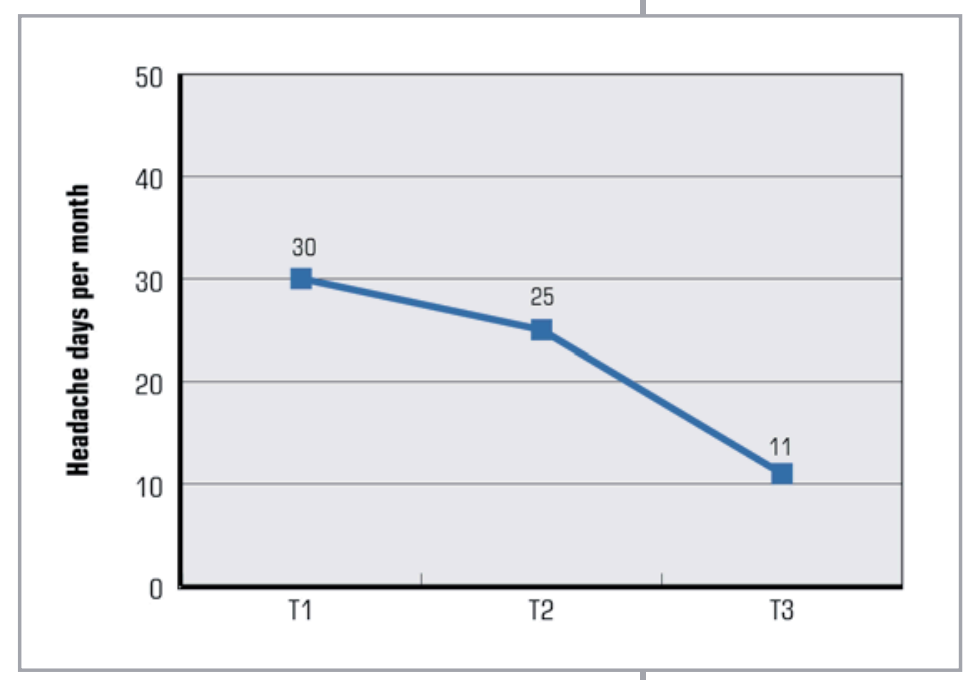

behavioral strategies. The importance of adhering to the prescribed medical therapy and communicating with the health care team was stressed. The patient was taught selfpacing and relaxation techniques aimed at helping her in reducing perceived stress and health-related and psychosocial anxiety. The importance of a healthy lifestyle in terms of eating, sleeping, and physical activity was pointed out.

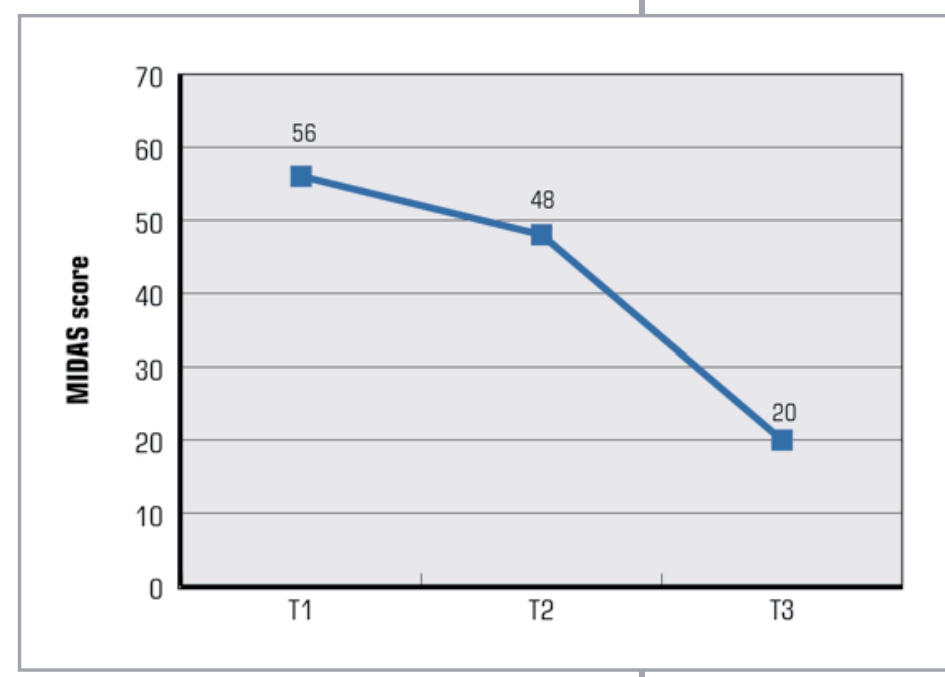

Three months after her last neurological visit, at a follow-up (T3), diaries revealed a better adherence and the patient communicated reduction of headache attacks in terms of intensity, frequency, and debilitation (11 HDPM; NRS = 7; MIDAS = 20), while referring an increased confidence in managing her headaches and a better perceived general health (Figures 1 and 2).
Figure 1. Headache frequency reduction $\mathrm{T} 1=$ first visit; $\mathrm{T} 2$ = first follow-up visit (after three months); $\mathrm{T} 3$ = second follow-up visit (after six months)
Figure 2. Headache disability reduction

MIDAS = Migraine Disability Assessment; $\mathrm{T} 1=$ first visit;

$\mathrm{T} 2=$ first follow-up visit (after three months);

$\mathrm{T} 3=$ second follow-up visit (after six months) 
Main questions a doctor should ask him/herself in this situation

- Does imaging show any previously undetected finding?

- Does the patient overuse NSAIDs?

- Have I assessed anxiety, depression, cognitive functioning, and disability?

- Does the patient need a more in-depth psychological analysis? Does helshe need to attend psychological sessions?

\section{DISCUSSION}

Different guidelines about headache have included several psychological and behavioral therapies, mostly as an auxiliary treatment, with various levels of recommendation $[5,6,13]$. Recommended psychological and behavioral interventions are, among others:

- Trigger management [13] (level III evidence, class B recommendation);

- Stress management [5,13];

- Cognitive-behavioral therapy $[6,13]$ (level II evidence, class B recommendation [13]);

- Relaxation techniques [6,13] (level II evidence, class B recommendation); and

- Patient education (level III evidence, class B recommendation) [13].

Psychological interventions for headache patients are advisable when at least one of the following criteria is met [9]:

- Personal factors that negatively influence headache management (e.g. beliefs about headache controllability and patient's own ability; personality traits);

- Poor adherence;

- Poor trigger management;

- Poor coping with headache;

- Medications overuse;

- Poor stress management; and

- Psychiatric comorbidity or other behavioral disorders.

It has to be underlined that psychological interventions in pain management are not substitutes of medical therapy, but they are intended to be used in conjunction with it. The considered case had a history of chronic headache and unsatisfactory past therapeutic results, characterized by a huge misuse of pain medications. Since the patient had previously and unsuccessfully taken therapy for migraine prevention, topiramate (level I recommendation) [6], then she was prescribed to switch to another medication, levetiracetam (off-label) $[6,14]$. In order to obtain better results, the patient had to stop overusing NSAIDs and was prescribed almotriptan (level I recommendation) [6] when needed. As she showed only partial improvement and was apparently still overusing NSAIDs, then it was proposed a behavioral approach in addition to the medical therapy and avoiding NSAIDs, in order to try and remove some obstacles to personal headache management.

This practice is compliant to literature and recommendations when some behavioral factors are relevant in a chronic headache patient $[6,9,13]$. In this case, excessive usage of medications, poor adherence, anxiety and some problems with coping headache and stress management were criteria that prompted the psychological referral.

Main strength of this report is that it presents a case treated using a model of care consistent with the biopsychosocial model and the most updated guidelines: interventions were tailored to the patient's features, consistently with literature about psychological headache management. Headache chronic patients that overuse medications present a obsessive-compulsive personality disorder: psychological management improves the patients' compliance to migraine prevention therapy and takes care of the psychological disorder aspect.

Main weakness is that, being this a singlecase study, it is not possible to demonstrate or quantify the contribution provided by psychological management.

\section{CONCLUSION}

All factors that determine headache onset, progression, and remission must be considered: when personal, behavioral, and environmental appear relevant, it is important to carefully assess these aspects and evaluate the opportunity of support the medical approach with psycho-social interventions, consistently with the biopsychosocial approach. This might help removing some obstacles in headache management and improving outcomes. 
Keypoints

- Medication Overuse Headache (MOH) is a Chronic Daily Headache caused by excessive use of pain medications

- The biopsychosocial model is a recent theoretical model underlining the interaction of physiological, psychological, and social factors that can help in understanding and treating chronic beadache

- Among the reasons for referring a headache patient to psychological support, there are not only the presence of major mental health and behavioral disorders (e.g. mood disorders, anxiety disorders, sleep disorder, eating disorders, and addictions), but also poor trigger management, poor stress management, and excessive use of medications

- According to the biopsychosocial model, a tailored psychological support, together with the most suitable medical therapy, may result in reduction of headache attacks in terms of intensity, frequency, debilitation and in a better perceived general health

\section{REFERENCES}

1. Pascual J, Colas R, Castillo J. Epidemiology of chronic daily headache. Curr Pain Headache Rep 2001; 5: 529-36; https://doi.org/10.1007/s11916-001-0070-6

2. Steiner TJ, Stovner LJ, Birbeck GL. Migraine: the seventh disabler. J Headache Pain 2013; 14: 1; https://doi.org/10.1186/1129-2377-14-1

3. Manack AN. Chronic Migraine: Epidemiology and Disease Burden. Curr Pain Headache Rep 2010; 15: 70-8; https://doi.org/10.1007/s11916-010-0157-z

4. International Headache Society. The International Classification of Headache Disorders, 3rd edition (beta version). Cephalalgia 2013;33:629-808; https://doi.org/10.1177/0333102413485658

5. Scottish Intercollegiate Guidelines Network. Diagnosis and management of headache in adults A national clinical guideline. Publication n. 107; 2008. Available from: www.sign.ac.uk/guidelines/ fulltext/107/index.html (last accessed January 2017)

6. Sarchielli P, Granella F, Prudenzano MP, et al. Italian guidelines for primary headaches: 2012 revised version.J Headache Pain 2012; 13: S31-S70; https://doi.org/10.1007/s10194-012-0437-6

7. Da Silva AN, Lake AE 3rd. Clinical aspects of medication overuse headaches. Headache 2014; 54: 211-7; https://doi.org/10.1111/head.12223

8. Pompili M, Di Cosimo D, Innamorati M. Psychiatric comorbidity in patients with chronic daily headache and migraine: a selective overview including personality traits and suicide risk. J Headache Pain 2009; 10: 283-90; https://doi.org/10.1007/s10194-009-0134-2

9. Nicholson RA. Chronic Headache: The role of the psychologist. Curr Pain Headache Rep 2010; 14: 47-54; https://doi.org/10.1007/s11916-009-0087-9

10. Nicholson RA, Houle TT, Rhudy JL, et al. Psychological risk factors in Headache. Headache 2007; 47: 413-26; https://doi.org/10.1111/j.1526-4610.2006.00716.x

11. Huguet A, McGrath PJ, Stinson J, et al. Efficacy of psychological treatment for headaches: an overview of systematic reviews and analysis of potential modifiers of treatment efficacy. Clin J Pain 2014; 30: 353-69; https://doi.org/10.1097/AJP.0b013e318298dd8b

12. Biagianti B, Grazzi L, Gambini $\mathrm{O}$, et al. Decision-making deficit in chronic migraine patients with medication overuse. Neurol Sci 2012; 33: S151-S155; https://doi.org/10.1007/s10072012-1071-4

13. Pryse-Phillips WEM, Dodick DV, Edmeads JG, et al. Guidelines for the nonpharmacologic management of migraine in clinical practice. CMAJ 1998; 159: 47-54

14. Verma A, Srivastava D, Kumar A, et al. Levetiracetam in migraine prophylaxis: a randomized placebo-controlled study in a rural medical institute in northern India. Clin Neuropharmacol 2013; 36: 193-7; https://doi.org/10.1097/WNF.0000000000000005 